\title{
Learning lessons from the common cold: How reducing parasite virulence improves coevolutionary optimization
}

\author{
John Cartlidge \& Seth Bullock \\ Informatics Research Institute, School of Computing, University of Leeds, Leeds, LS2 9JT \\ Tel: +44 (0)113233 5322, Fax: +44 (0)113233 5468, Email: \{johnc, seth\}@comp.leeds.ac.uk
}

\begin{abstract}
Inspired by the virulence of natural parasites, a novel approach is developed to tackle disengagement, a detrimental phenomenon coevolutionary systems sometimes experience [1]. After demonstrating beneficial results in a simple model, minimumcomparison sorting networks are coevolved, with results suggesting that moderating parasite virulence can help in practical problem domains.
\end{abstract}

\section{INTRODUCTION}

Artificial coevolution can be divided into two main categories, cooperative and competitive coevolution. In cooperative coevolution individual fitness is determined by a series of collaborations with others [2]. Cooperative coevolution is often used to tackle problems that can be naturally decomposed, with each individual representing a sub-part.

Competitive coevolution, the focus of this paper, is the most commonly used class of coevolutionary algorithm [3], [4], [5], [6]. In competitive coevolution, individual fitness is determined by a series of competitions with others. Individuals typically represent complete solutions that are gradually refined throughout the run.

Although natural coevolution involves multiple populations, this is not required of artificial coevolution. One population may be engaged in competitive self-play, where individuals are assessed by opponents from within the population, or there could be intra-population cooperation [7], [8].

The fundamental difference between coevolutionary algorithms and standard evolutionary algorithms is the way in which fitness is evaluated. Standard evolution incorporates an absolute fitness function, whereas coevolution utilises the concept of a relative fitness measure.

Absolute fitness measures are static functions measuring performance against a yardstick external to the simulation environment. When trying to evolve a fast car design, for example, an absolute function might make fitness proportional to top speed.

Relative fitness measures are dynamic functions sensitive to conditions within the simulation environment. When coevolving a fast car, a relative fitness measure might rate designs through comparison with their coevolving competitors, e.g., racing a sample of designs against each other and awarding fitness proportional to the number of races won. Relative fitness measures are especially useful when absolute performance indicators are difficult to define.

It is important to separate this distinction from that between explicit and implicit fitness functions. Explicit fitness functions are mathematical equations clearly defined to determine the fitness of each individual, e.g., fitness $\propto$ speed $\times$ safety. Implicit fitness functions (usually used with steady-state models) allow an agent's fitness to arise directly from interactions with the environment, e.g., reproduction occurs once 100 successful journeys have been made.

Although coevolution offers a powerful alternative to standard evolution's static landscape, it suffers from several problems. Populations may evolve to exploit the idiosyncrasies of their opponents, leading to overspecialisation and fragility. Moreover, coevolutionary systems are notoriously difficult to analyse - it is hard to tell whether sustained competition is leading to smooth directional evolutionary progress, to noisy fits and starts, or to endless cycling through different evolutionary unstable strategies. In this paper, however, we concentrate on the related problem of disengagement [1].

As coevolutionary algorithms assess individuals through pitting them against opponents, there arises the problem of ensuring that opponents are worth beating. Disengagement occurs when a population faces opponents that are either all too easy or too difficult to beat. In such a situation all individuals will score identically and the fitness gradient driving coevolution will disappear leading to a period of degrading evolutionary drift. Disengagement will be particularly pronounced when one population has an advantage over the other. For instance, at the outset of coevolution, successful evasion may be much easier than successful pursuit [7]. We can expect the initial population of evaders to immediately disengage from the coevolving population of pursuers.

The remainder of the paper is arranged as follows. Section II defines an abstraction of parasite virulence inspired by natural host-parasite systems. In Section III we use a simple model to demonstrate the benefits of encouraging reduced virulence with respect to disengagement. The real-world problem of designing minimumlength sorting networks is approached in Section IV, with experimental results suggesting that moderating virulence can aid coevolutionary optimization in practical problem domains. Finally, Section V concludes, discussing open questions and possible future work. 
a) Maximum

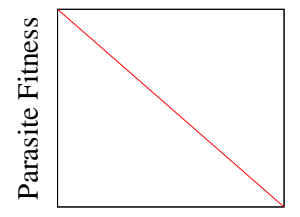

Host Score b) Moderate

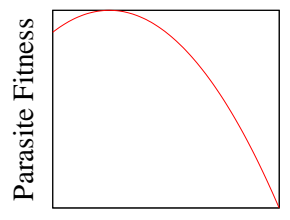

Host Score

Figure 1: Fitness Functions Encouraging Maximally and Moderately Virulent Parasites.

\section{PARASITE VIRULENCE}

\section{A. Virulence in Nature}

Natural parasites exhibit a wide range of virulence ${ }^{1}$ levels. Parasites such as malaria are deadly, whilst others, such as the common cold, are often a mere nuisance. Parasite virulence evolves to maximise parasite progeny, and in order to ensure survival long enough to reproduce, it is certainly not always in the best interest of a parasite to be as virulent as possible [10].

Whilst a parasite gains from using a host's resources, incapacitating a host can greatly reduce a parasite's chances of reproducing. For this reason, a parasite's virulence and dispersal distance are strongly linked [11]. A parasite that greatly relies on the mobility of a particular host is likely to have relatively mild virulence. In contrast, parasites that are easily transmitted from one host to another, irrespective of host mobility, are likely to remain highly virulent. Using mosquitoes as a transmission vector, malaria can afford to fatally damage hosts without hindering propagation. Indeed, a host rendered unconscious is more susceptible to mosquito bites, ultimately aiding transmission. In stark contrast, as the common cold spreads via host-host contact, it benefits from keeping hosts active and sociable.

\section{B. Virulence in Artificial Coevolution}

Artificial coevolutionary algorithms generally employ maximally virulent parasites [3], [6]. It seems widely (although tacitly) accepted that this is for the best since parasites that hinder sub-optimal hosts are thought to provide a powerful selection pressure ensuring that only the strongest host strategies survive (for an alternative disease-based approach to models of coevolution see [12]).

However, we propose that encouraging reduced parasite virulence will aid the coevolutionary process by preventing disengagement. By punishing those parasites that gain too great an advantage over their hosts, we may be able to avoid scenarios in which one population outstrips the other to such an extent that the relative fitness

\footnotetext{
${ }^{1}$ Defined as "parasite-mediated morbidity and mortality in infected hosts" [9].
}

\begin{tabular}{|c|c|c|c|c|c|c|c|c|c|c|c|c|}
\hline \multirow[b]{2}{*}{ SUM } & 1 & 0 & 1 & & 0 & 1 & 0 & 1 & 0 & 0 & 1 & \multirow{2}{*}{$\begin{aligned} & \text { Haploid } \\
= & 5\end{aligned}$} \\
\hline & 1 & & 1 & & & 1 & & 1 & & & 1 & \\
\hline \multirow{2}{*}{ OR } & 1 & 0 & 0 & & 1 & 1 & 0 & 0 & 1 & \begin{tabular}{|l|l}
0 \\
\end{tabular} & 1 & \multirow{2}{*}{ Diploid } \\
\hline & \begin{tabular}{|l|}
0 \\
\end{tabular} & 1 & 0 & & 1 & 0 & 0 & 0 & 1 & \begin{tabular}{|l|l}
1 \\
\end{tabular} & 1 & \\
\hline SUM & 1 & 1 & & & 1 & 1 & & & 1 & 1 & 1 & $=7$ \\
\hline \multirow{3}{*}{$\begin{array}{l}\text { OR } \\
\text { OR }\end{array}$} & 0 & 0 & 1 & & 0 & 0 & 1 & 0 & 1 & 1 & 0 & \multirow{3}{*}{ Triploid } \\
\hline & 0 & 1 & ( & & 0 & 1 & 0 & 0 & 0 & 1 & 1 & \\
\hline & 1 & 1 & ( & & 0 & 0 & 0 & 1 & 0 & 1 & 0 & \\
\hline SUM & 1 & 1 & t & & & 1 & 1 & 1 & 1 & 1 & 1 & $=9$ \\
\hline
\end{tabular}

Figure 2: Scoring process for a simple counting ones game. Each loci with at least one 1-allele contributes +1 to the sum.

function fails to discriminate amongst each population, resulting in coevolutionary drift.

In order to test this hypothesis, we must define scenarios that promote maximum and moderate parasite virulence in the context of artificial coevolutionary systems. Pressure for maximal virulence is provided by a function in which a parasite's fitness is reciprocal to that of its hosts (Figure 1, left). In contrast, pressure for moderate virulence can be implemented by awarding maximum parasite fitness when hosts secure some modicum of success, defined here as $25 \%$ (Figure 1, right). Any deviation from this optimal level reduces the fitness of the parasite. Parasites selected for moderate virulence, like the common cold, are punished if they inflict too much damage on the host.

\section{MODERATING PARASITE VIRULENCE IN A MINIMAL SUBSTRATE}

\section{A. The Experiment}

Inspired by the minimal substrate that Watson and Pollack use to explore various coevolutionary phenomena, we chose to demonstrate the effect of moderating parasite virulence on a coevolutionary version of the counting ones problem [1]. Two populations were coevolved via asexual reproduction with the probability of bit-flip mutation at 0.05 . Tournament selection was employed with tournament size 5 . One population contained 25 haploid hosts, each represented by a binary genome with 100 loci. The other population contained 25 diploid parasites represented by two binary strings of length 100 . At the outset, all genomes were intialised to 0 at each loci.

At each generation, each host is paired with a randomly chosen parasite against which it plays a simple game: whichever player has more loci at which the 1allele is present wins (see Figure 2). Hosts and parasites compete in this way (with a new random partner for each game) for $N$ games, and are each allocated a score out of $N$ (ties are worth half a win). The fitness of an individual is related to the percentage of contests won against the sample of opponents. Fitness thus depends upon which opponents are chosen. Individual $a$, with 
many 1-alleles, may be less fit than another individual $b$, with fewer, if the sample of individuals chosen to play $a$ is much stronger. Notice that in general diploid parasites have a clear advantage over haploid hosts since they have two alleles at each loci. Whereas an average haploid individual will have 1-alleles at 50 of its loci, an average diploid individual will have at least one 1-allele at 75 loci. By giving parasites an advantage in this way, we hoped to encourage disengagement: the greater the advantage one population has, the greater the chance of every individual in the advantaged population outperforming every individual in the disadvantaged population.

In a second experiment, this asymmetry was increased by employing triploid parasites. Under these conditions, the average parasite will posses at least one 1-allele at $87.5 \%$ of its 100 loci.

For each experiment we compared coevolutionary performance with parasites encouraged to be maximally virulent (fitness proportional to games won) to that with parasites encouraged to be only moderately virulent (Figure 1, right). In all experiments, host fitness is proportional to games won.

\section{B. Results}

Results from experiment 1 featuring diploid parasites are displayed in Figure 3. Though these graphs were handchosen, they are representative. Under pressure for maximal virulence both populations progress rapidly but then stall. Between roughly generation 75 and 450 they disengage and degrade to their respective baseline levels of performance $(50 \%$ and $75 \%$ ). By favouring reduced parasite virulence, we encourage the populations to remain engaged, and thus avoid periods of degrading drift.

Results from experiment 2 featuring triploid parasites are displayed in Figure 4. Though these graphs were also hand-chosen, they too are representative. Under pressure for maximal virulence parasites disengage immediately and forever. In contrast, every run using moderate virulence maintains population engagement despite the underlying asymmetry.

\section{Discussion}

Results clearly demonstrate that moderating parasite virulence can prevent coevolutionary disengagement in asymmetric scenarios. Furthermore, the greater the inherent asymmetry, the greater the effect moderating virulence has upon results.

The asymmetry imposed in this model gave the diploid and triploid parasites an advantage over the coevolving haploid population. Eventually, purely by chance, one would expect all the individuals from the diploid and triploid populations to beat all those in the haploid population.

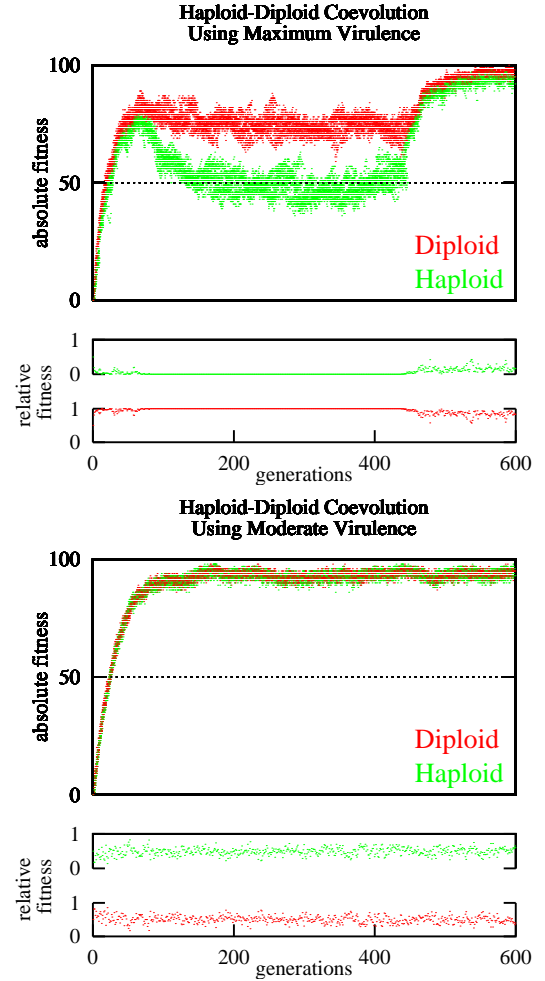

Figure 3: Typical diploid vs. hapoloid coevolution under maximal and moderate virulence conditions. The large graphs display the absolute fitness of each individual over 600 generations of coevolution, while the smaller graphs show the variation in mean population relative fitness over the same period. Disengagement occurs when the average relative fitness of a population is either 1 or 0 , e.g., between generations 75 and 450 in the top graph. Each individual is rated against a sample of $N=5$ opponents.

This is exactly what is observed under conditions promoting maximal virulence. Within one or two generations, disengagement occurs. Although coevolutionary selection pressure is removed as soon as the populations disengage, mutation bias pushes the populations towards the expected average percentage of 1-alleles. The haploid and diploid populations tend towards $50 \%$ and $75 \%$ respectively. Random variation ensures that these populations re-engage periodically, entering a brief arms race that is always won by the advantaged parasites who may achieve disengagement once more, inducing a fresh period of degrading drift. During disengagement triploid populations tend towards $87.5 \%$ 1-alleles, ensuring that once disengagement occurs, the populations will remain disengaged until a large (and hence very unlikely) number of beneficial mutations occurs.

Under moderate virulence conditions, the parasite population does not quickly outstrip the hosts. Unlike the maximally virulent parasites, moderate parasites are not rewarded for beating as many opponents as possible. Although the first generation of diploid/triploid offspring will on average contain many more 1-alleles than 

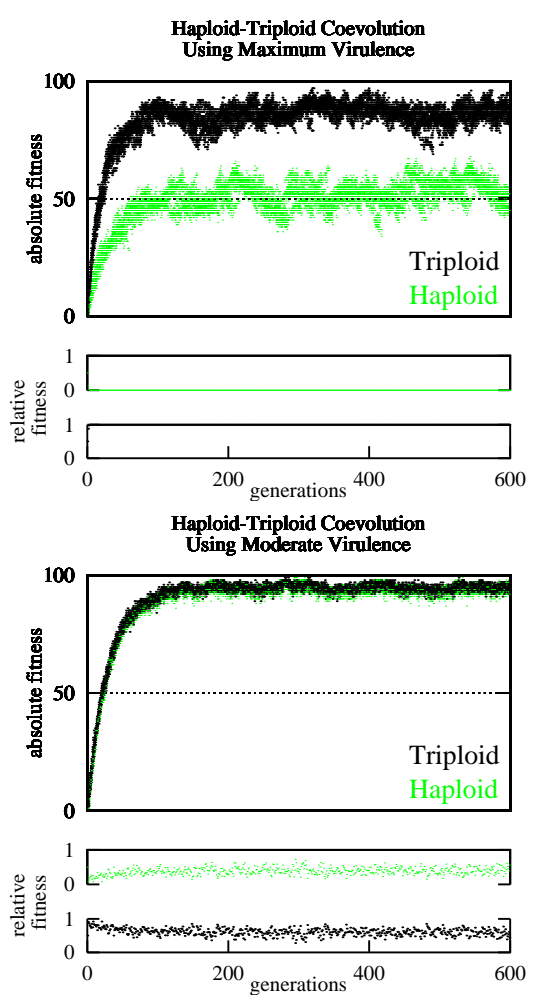

Figure 4: Typical triploid vs. hapoloid coevolution under maximal (top) and moderate (bottom) conditions. Details as for Figure 3.

the haploid population, those diploid/triploid parasites that beat all opponents will actually be less fit than those parasites that lose a small percentage of contests. In this way, the parasite population is rewarded for resisting disengagement. The continued engagement and associated selection pressure encourages hosts to evolve to a greater level than would otherwise be possible. Interestingly, the moderate parasites gain from this relationship too, also evolving to a greater standard than their maximally virulent cousins.

\section{COEVOLVING MINIMUM LENGTH SORTING NETWORKS}

The evidence suggested by Figures 3 and 4 strongly supports the case that moderating virulence can reduce disengagement effects and thus aid coevolutionary optimization. However, is disengagement a regular phenomenon that occurs in real-world, optimization problems, or is it merely an artifact of the simple set-up used in Section III? In an attempt to answer this question, the coevolution of minimum-length sorting networks was chosen as a typical domain. Although it is difficult to determine whether the design of sorting networks is an average problem, it is certainly popular [3], [5], [6]. The aim is to discover the smallest network of comparisons that can sort, into numerical order, any input list containing a specific number of elements, where comparisons exist in the form if $a>b$ then swap, else do nothing.

\section{A. Background}

The seminal work of Hillis proved by example how simulated coevolution can be applied to practical optimization problems [3]. As a problem domain, Hillis chose to design minimum-comparison sorting networks for sixteenelement inputs (for a further discussion see [13]). The design of minimum-comparison sorting networks has a long and competitive history, resulting in the current best known of sixty comparisons to sort sixteen elements. This was achieved by hand in 1960 by M. W. Green [14].

Hillis evolved the sorting networks on a static fitness landscape, the fitness of each network determined by its ability to sort a random sample of input lists. This approach resulted in a best network of 64 comparisons. Hillis found that two factors were preventing the evolution of shorter networks. Primo, the classical problem of local optima made it difficult for the system to progress once a reasonable solution had been found. Secundo, the test process proved inefficient-after relatively few generations, most of the random lists were sorted fully by the majority of networks.

To compensate for these problems, Hillis allowed the test cases to coevolve with the sorting networks, giving them complementary (reciprocal) fitness functions, thus implementing an artificial (maximum virulence) hostparasite system. This allowed the test lists to evolve, punishing the weaknesses of the evolving networks and encouraging them to move on from local optima, whilst dramatically reducing the number of redundant tests that were easy to sort. Through this approach, Hillis discovered a minimum sorting network of only 61 comparisons, a much better solution than previously found and only one comparison longer than the best-known solution.

\section{B. Using Moderated Virulence: The Experiment}

Currently, the minimum-length comparison network known to sort 13-element lists is 45 . For historical reasons $^{2}$ this was chosen as our problem domain. We were not interested in the solutions discovered, but in the differences in performance that varying parasite virulence might induce. Host networks consisted of 45 pairs of integers, with each pair representing list elements to be compared and, if necessary, swapped. Host mutation occurred at each loci with probability 0.02 , producing a random integer in the range 1 to 13 . Parasites each contained 40 unsorted 13 -element lists. In order to preserve lists as permutations of the integers 1 to 13 , parasite mutation consisted of swapping two elements of the list. This occurred at each loci with probability 0.05 .

Both parasite and host population sizes were identical, with each host attempting to sort exactly one parasite.

\footnotetext{
${ }^{2}$ The shortest network currently known for this problem was discovered using a coevolutionary algorithm [5].
} 

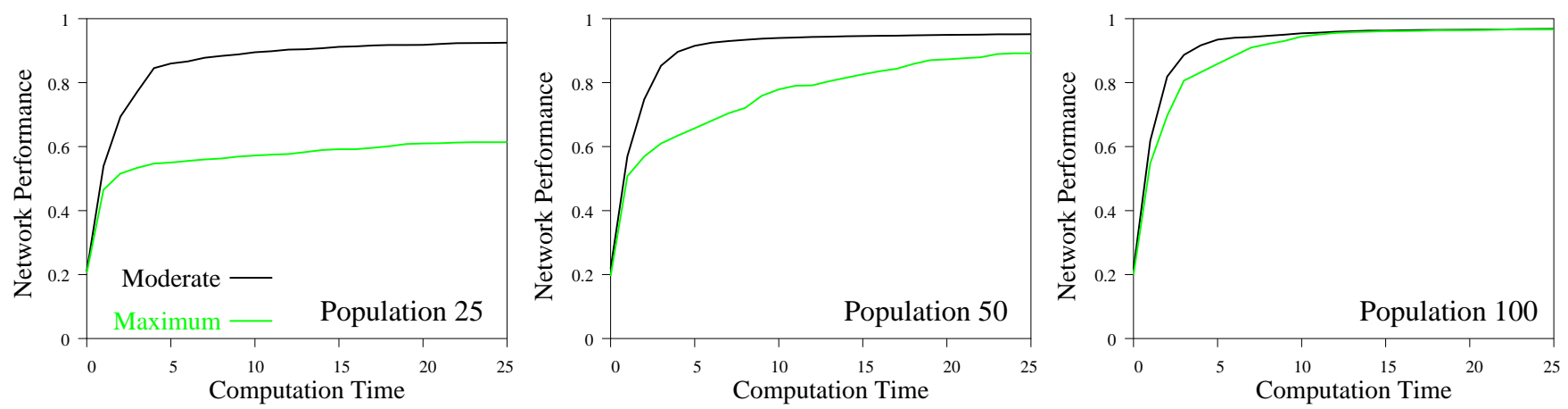

Figure 5: The average performance $(N=30)$ of evolved sorting networks for 13-element input lists over coevolutionary time. The graphs show the benefit of moderating virulence greatly increases as population sizes fall. Computation Time is proportional to the total number of computational steps taken.

Both hosts and parasites were asexual. Tournament selection was used, with tournament size 7 .

Host fitness was calculated as proportional to the number of parasite lists that were completely sorted. Reciprocally, parasites were rewarded for possessing lists that were unsorted. Parasite scores were normalised relative to the highest scoring parasite in the population before being mapped to fitness via either a function favouring maximal or moderate virulence.

In order to collect accurate statistics, 30 runs were carried out for each condition with population sizes ranging from 25 to 100 . An advantage of this problem domain is that an absolute, objective fitness measure of hosts is possible - networks are given every possible input to sort ${ }^{3}$ with the percentage of correctly sorted lists determining an absolute fitness performance. A host network that can sort $100 \%$ of all possible inputs is an optimal network. For each population size, the performance level achieved under moderate and maximal virulence conditions was compared by calculating the mean absolute performance of the best host in each generation.

\section{Results}

Figure 5 displays the mean absolute performance of the best network evolved against computational time. ${ }^{4}$ It can clearly be seen that using moderately virulent parasites leads to improved performance when the population size is only 25. As population size grows, the magnitude of this improvement steadily decreases.

These results were found to be insensitive to variation in mutation rate, tournament size and, to a great extent, problem complexity. When 8-element sorting net-

\footnotetext{
${ }^{3}$ The set of input lists can be calculated efficiently by using the zero-one principle: 'a network can sort every input list if and only if it can sort every binary input list' [14].

${ }^{4}$ In order to make comparisons valid across variation in population size, we ran coevolution for the same amount of computational time rather than the same number of generations. i.e., the greater the population size, the smaller the number of generations per unit computation time.
}

works were evolved, the results were qualitatively similar, with moderate virulence increasingly outperforming maximum virulence as population sizes fall. However, the reduction in problem complexity allowed both virulence schemes to perform well, greatly reducing the actual magnitude of the advantage displayed by moderate virulence.

\section{Discussion}

In Section III we demonstrated that moderating parasite virulence prevents disengagement in a simple problem domain. Is it thus reasonable to suggest, given Figure 5, that moderating virulence is reducing disengagement in the real-world problem domain of minimum-length sorting networks and that this is responsible for improved performance?

The fact that, as population size falls, the benefit of moderating virulence increases is what we would expect if moderate virulence was indeed helping to stop disengagement since disengagement becomes progressively more likely as population sizes fall. Considering only stochastic effects, it is intuitive that the greater population size becomes, the less likely it is that all the individuals in one population will beat all the individuals in another population. At the other extreme, two populations of size one will always be disengaged.

The results are very suggestive, but are certainly not conclusive. Evolving minimum-length sorting networks is a very complex problem domain, making it very difficult to analyse. However, we would expect the listsorting problem to exhibit the kind of asymmetry that led to disengagement in the counting-ones problem. Being a challenging set of lists is much easier, at least at the outset of coevolution, than being an accomplished list sorter. Could this asymmetry account for the relatively slow progress made by conventional coevolution? If this type of initial asymmetry is a feature of many coevolutionary optimization problems, moderating parasite virulence in some way could turn out to be an approach 
with wide application.

\section{CONCLUSIONS}

The focus of this paper has concerned the problem of coevolutionary disengagement. Disengagement has been defined here as occurring when every member of one population is better than every member of the coevolving population. It leads to a loss of selection pressure and causes populations to drift, disrupting evolutionary progress.

Exploiting the fact that parasite virulence is known to vary in natural systems, we have identified a means of abstracting virulence as a tool that can be used in artificial systems. Using a minimal substrate we have shown that moderating virulence can reduce disengagement in some simple systems. Moreover, we have demonstrated that moderating virulence can improve coevolutionary optimization in the real-world domain of minimum-length sorting networks.

This bodes well for the future of simulated coevolution. If disengagement is a problem hindering coevolution in many domains then moderating parasite virulence could potentially be a practical, versatile tool, to aid coevolution optimization.

\section{A. Future Work}

Throughout this paper, moderate virulence has been encouraged by employing a particular fixed fitness function (Figure 1). This has been useful to demonstrate the relationship between virulence and disengagement. However, since the degree of engagement experienced by two coevolving populations will typically vary over the course of a coevolutionary run, in general it will not be profitable to encourage a particular, fixed level of virulence. ${ }^{5}$ We intend to construct a metric with which to measure the degree of disengagement within a coevolving system. This can then be used to modulate both the parasite and host fitness functions such that when populations are fully engaged, maximal virulence is favoured, pushing coevolution onwards, whereas when populations are fully disengaged, pressure for moderate virulence coheres them. Hopefully this will further improve coevolutionary success.

\section{Acknowledgments}

The authors would like to thank Jason Noble, Dave Harris and Tom Carden for many hours of discussion and also Richard Watson, Sevan Ficici and the three anonymous reviewers for their time and useful comments.

\section{References}

[1] R. A. Watson and J. B. Pollack. Coevolutionary dynamics in a minimal substrate. In Spencer et al. [16], pages 702-709.

\footnotetext{
${ }^{5}$ E.g., pressure on parasites to remain only moderately virulent, may be responsible for the failure of hosts to achieve perfect solutions in the counting-ones problem.
}

[2] R. P. Wiegand, W. C. Liles, and K. A. De Jong. An empirical analysis of collaboration methods in cooperative coevolutionary algorithms. In Spencer et al. [16], pages 1235-1245.

[3] W. D. Hillis. Co-evolving parasites improve simulated evolution as an optimization procedure. Physica D, 42:228-234, 1990

[4] C. D. Rosin and R. K. Belew. Methods for competetive coevolution: Finding opponents worth beating. In L. J. Eshelman, editor, Proceedings of the Sixth International Conference on Genetic Algorithms, pages 373-380. Morgan Kaufmann, San Francisco, CA, 1995.

[5] H. Juillé. Incremental co-evolution of organisms: A new approach for optimisation and discovery of strategies. In Morán et al. [18], pages 246-260.

[6] B. Olsson. Optimisation using a host-parasite model with variable-size distributed populations. In Proceedings of the 1996 IEEE International Conference on Evolutionary Computation, pages 295-299. IEEE Press, 1996.

[7] D. Cliff and G. F. Miller. Coevolution of pursuit and evasion II: Simulation methods and results. In Maes et al. [15], pages 506-515.

[8] R. A. Watson and J. B. Pollack. Symbiotic combination as an alternative to sexual recombination in genetic algorithms. In M. Schoenauer, K. Deb, G. Rudolph, X. Yao, E. Lutton, J. J. Merelo, and H.P. Schwefel, editors, Parallel Problem Solving from Nature 2000, PPSN VI. Springer-Verlag, 2000.

[9] B. R. Levin. The evolution and maintenance of virulence in microparasites. Emerging Infectious Diseases, 2(2), 1996.

[10] C. Combes. Ethological aspects of parasite transmission. The American Naturalist, 138(4), 1991.

[11] C. C. Maley. A model of the effects of dispersal distance on the evolution of virulence in parasites. In Brooks and Maes [17], pages 102-108.

[12] J. Epstein and R. Axtell. Growing Artificial Societies: Social Science from the Bottom Up. Brookings Institute Press/MIT Press, Washington, DC; Cambridge, MA, 1996.

[13] M. Mitchell. An Introduction to Genetic Algorithms. MIT Press, Cambridge, MA, 1996.

[14] D. E. Knuth. The Art of Computer Programming - Sorting and Searching, volume 3. Addison Wesley, 1973.

[15] P. Maes, M. Matarić, J.-A. Meyer, J. Pollack, and S. W. Wilson, editors. From Animals to Animats 4: Proceedings of the Fourth International Conference on Simulation of Adaptive Behavior. MIT Press, Cambridge, MA, 1996.

[16] L. Spencer, E. D. Goodman, A. Wu, W.B. Langdon, H., M. Gen, S. Sen, M. Dorigo, S. Pezeshk, M. H. Garzon, and E. Burke, editors. GECCO 2001: Proceedings of the Genetic and Evolutionary Computation Conference. Morgan Kaufmann, 2001.

[17] R. A. Brooks and P. Maes, editors. Artificial Life IV. MIT Press, Cambridge, MA, 1994.

[18] F. Morán, A. Moreno, J. J. Merelo, and P. Chacón, editors. Advances in Artificial Life: Third European Conference on Artificial Life (ECAL '95), volume 929 of Lecture Notes in Artificial Intelligence. Springer, Berlin, 1995. 